\title{
An Automatic Dual Axis Sun Tracker and Temperature Monitoring System for Novel Solar Dish Concentrator
}

\author{
Krishna Kumar.N ${ }^{\mathrm{a}^{*}}$, Venkat Subramaniam ${ }^{\mathrm{b}}$ \\ ${ }^{a}$ Ph.D Scholar, Department of Electronics, PSG College of Arts \& Science, Coimbatore, 641 014, India \\ ${ }^{b}$ Associate Professor, Department of Electronics, PSG College of Arts \& Science, Coimbatore, 641 014, India
}

Received: 07 May 2017; Accepted: 11 September 2017; Published: 08 January 2018

\begin{abstract}
Nonpolluting, renewable energy can be harnessed from the sun using solar thermal concentrator and photovoltaic systems. A novel asymmetric oblique compound conical solar concentrator was designed and constructed with automatic solar tracking system. The experimental study of the developed system was carried out to inspect the efficiency of the designed solar concentrator and automatic azimuth and altitude tracking. In this solar tracking, the RTC (Real Time clock) based active solar tracking method is used to track the sun in order to attain the maximum temperature. A simple microcontroller based control system is used to control this open loop solar tracking system, further the concentrated thermal power was measured using both the K-type temperature sensor and thermometer gun. The temperature obtained at the receiver of solar concentrator is sent to the measurement unit using GSM wireless module available at remote location. The monitoring unit receives the temperature values and recoded them regularly. Besides, the maximum average solar thermal power of the system was observed to be during $10.00 \mathrm{AM}$ to $3.00 \mathrm{PM}$. This temperature is useful for various heating applications.
\end{abstract}

Index Terms: RTC, Sun Tracker, Ray Trace, GSM Module, Solar Concentrator, Microcontroller.

(C) 2018 Published by MECS Publisher. Selection and/or peer review under responsibility of the Research Association of Modern Education and Computer Science.

\section{Introduction}

Non-renewable energy sources such as fossil fuels are finite and will be running down the timeline in future. Renewable energy sources are clean and least environmental polluting than usual energy sources such as coal, oil, and gas etc. There are different automatic solar tracking systems is used in the different solar concentrators.

* Corresponding author. Tel.: +91-9894888862;

E-mail address: nkrishnamsc@gmail.com 
In solar concentrators using tracking a simple timing device is used to adjusting azimuth angle by three times at three fixed position east, south and west from the morning of the day, noon of the day and afternoon of the day. The solar tracking system increases daily collection of radiation and the annual collectible solar radiation by 92\% [1]. The PLC based dual axis sun tracking for parabolic solar concentrator was designed and constructed in order to overcome the continuous tracking and manual operations. PLC is used to control the overall tracking mechanism and water reaches only $90{ }^{\circ} \mathrm{C}$ inside the receiver tube [2]. Chong and Wong developed a general formula for On-Axis Sun-Tracking System. The tracking accuracy of solar concentrator is also studied. The most common method of azimuth elevation and tilt tracking mechanism are used in the solar tracking in order to orient the concentrator in sun's position at all the times. Solar tracking method plays a main role to make sure that the solar concentrator can collect maximum solar irradiation. Normally, the photo sensor signal is used as a feedback signal to track the sun at all the time [3]. The design of PLC (Programmable Logic Control) based dual axis solar tracking system uses an open loop control method. Various controlling functions are implemented using software which is stored into PLC's memory and these functions are used to track the sun and orient the solar concentrator. A maximum temperature of only $93{ }^{\circ} \mathrm{C}$ was obtained [4] in spite of enough instrumentation and technology. Abouzied developed programmable logic array based closed loop solar tracking system. It includes PLA (Programmable Logic Array), 8/6 four-phase RSC (Reluctance Stepper Motor), two separate sensing cells and power supply of 220 VDC. Two separate sensing cell outputs are fed to the PLA control circuit in order to determine the subsequent angular position of sun. It was mainly used for stand-alone system in remote areas. The power consumption of this system and as well the cost seems to be high [5]. The output of the LDR (light dependent resistor) sensor was given to closed loop programming method in order to track the sun. The efficiency of the system depends on design of the shadow mask of LDR sensors or photodiode whichever was preferred. The entire solar tracking process and temperature measurements are transmitted to the computer via zigbee wireless module and monitored by the computer. The advantage of the system is low cost and low power. The drawback of using zigbee module for data transmission is the range which is maximum of around 100 meters distance and LDR fails to predict the precise location of the sun especially under cloudy conditions of a day [6]. A PLC based active and passive solar tracking system is used for a large dish solar concentrator system. It included four electro-optical sensors which are used to detect the light intensity. The output of optical sensor was based on the light intensity. When the output meets the requirements of the system, then the system turn into passive solar tracking mode otherwise, it will run in active solar tracking mode. The accuracy of this system depends on the sensor and software coding. The efficiency of a concentrator could therefore be improved when the concentrator and its instrumentation has the good combination of active tracking and passive tracking [7]. A solar tracking system based on computer image processing of a sensor based shadow arrangement when investigated was found to be independent of the geographical place and stage alignment of equipment. This solar tracking system included computer, AVR Microcontroller, DC motor and Photo sensor. The main drawback of this system is portability and cost [8]. Hamed and El-Moghany designed and implemented FLC (Fuzzy Logic Control) based on FPGA for solar tracking mechanism and tested using Matlab/Simulink software tool. This solar tracking system uses two LDRs placed at the east-west direction or north-south direction in order to detect the sun's position by receiving light intensity. LDR sensor was understood to be more reliable, for this low cost and low power consuming system. But the sensing accuracy is less for cloudy conditions, dust on the sensor and physical damages on it [9]. In $\mathrm{PV} / \mathrm{T}$ systems, automation is found to be a requirement in order to orient towards the sun. An AT89C52 microcontroller based solar tracking system is used to control the entire automation. This system automation is also dependant on LDR sensor, which is used to detect and provide output based on light intensity on it. The disadvantage of this solar tracking system is that it goes to idle mode when cloudy or under dark conditions [10]. MPPT (Maximum Power Point Tracking) for a PV system under different solar irradiation conditions and temperature is simulated using an artificial intelligent neural network. The effectiveness of this system is better. Maximum power point tracking system requires more power for scanning voltage and orienting the PV panel [11].Considering all these studies on tracking concentrators and understanding the need of a cost effective, low power consuming, portable, and good outcome based system design was on top priority in my work, which is 
independent of non-linearity at the component -level for various environmental conditions. Also the RTC included in the system determines the position of the sun at specific times and orients the concentrator subsequently making it a real time system.

\section{Mechanical Design of the Solar Dish and Equations}

The proposed dish concentrator system was designed using CAD tool (Computer Aided Drawing). The elliptical based entry aperture of the dish is used to receive the maximum radiation on the dish concentrator and this solar radiation converges on the receiver. The aspect ratio of the each mirror used is 1:1 inch. The supporting system of the entire system is low cost, portable and easily assembled. The entry aperture designed has $138.0 \mathrm{~cm}$ major axis and $122.0 \mathrm{~cm}$ minor axis. Top and bottom view of the dish is shown in figure $1(\mathrm{~A})$ and (B). The major axis of the dish is $\mathrm{x}$ and minor axis of the dish is $\mathrm{y} . \mathrm{c} 1, \mathrm{c} 2$, and $\mathrm{c} 3$ are circumferences of the compound oblique cone dish. The depth of the dish is represented as d. The focal length (f) of the dish is 109.0 $\mathrm{cm}$. The area of an elliptical based entry aperture is calculated using the length of the major axis (half of $\mathrm{x}$ ) and the length of the minor axis (half of y).

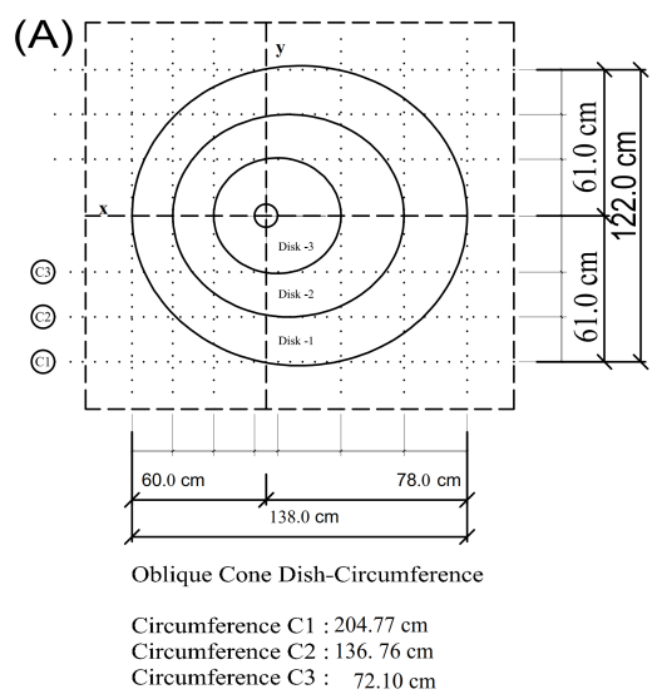

(B)

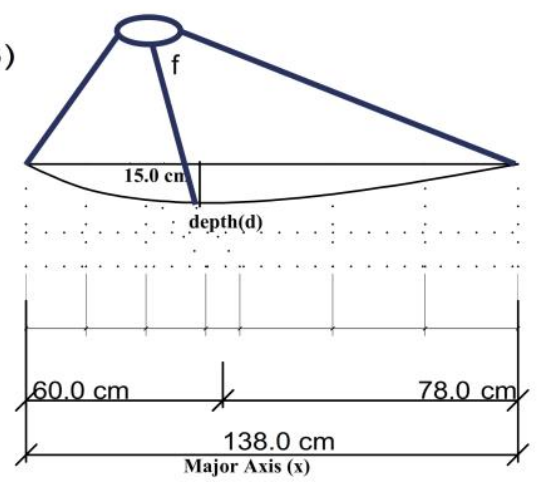

Fig.1. (a) Top View; (b) Front View 
The following formula is used to calculate the area of an elliptical based entry aperture,

$$
\mathrm{A}=\pi \times a \times b
$$

The half of the major and minor axis is ' $a$ ' and ' $b$ ' respectively. The ' $A$ ' represents an area of an ellipse. Circumference formula of an ellipse approximated by Ramanujan is,

$$
\mathrm{P}=\pi[3(a+b)-\sqrt{((3 a+b)(3 b+a))}]
$$
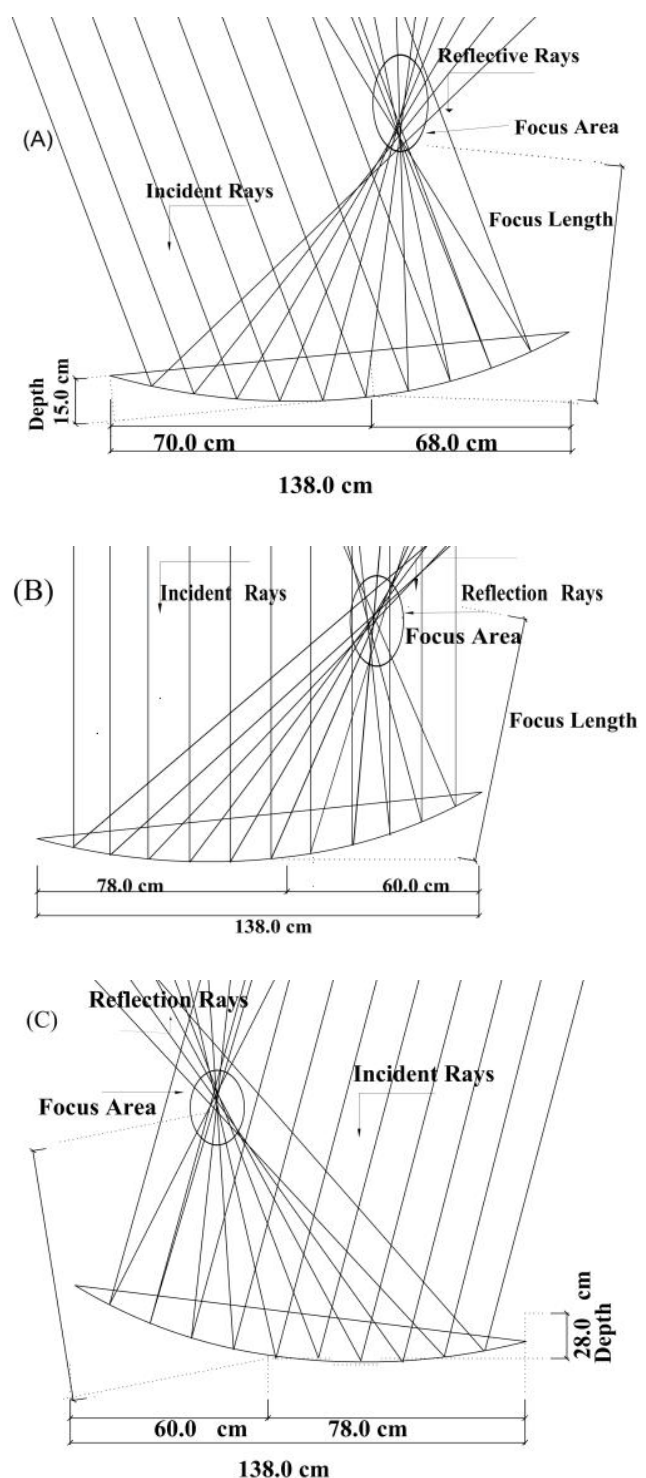

Fig.2. Ray Tracing (a) 11.00 am; (b) 12.00 pm; (c) 13.00 pm 
' $\mathrm{P}$ ' is the perimeter of an elliptical based entry aperture. The ray trace was done with the help of AutoCAD software tool. Fig. 2(A), (B) and (C) shows the ray trace for different time interval $11.00 \mathrm{am}, 12.00 \mathrm{pm}, 13.00$ $\mathrm{pm}$ respectively. The focus will vary within the elliptical receiver. The major axis of the receiver is $22.5 \mathrm{~cm}$ and minor axis of the receiver is $6 \mathrm{~cm}$.

The ray trace has been done for a designed dish concentrator model using AutoCAD software tool. For different timing, ray trace has shown in the above figure. According to the ray trace design, the solar tracking concentrator was tested and observations were recorded.

\section{Hardware and Software Description of the RTC Based Solar Tracker}

A simple stepper motor based mechanical structure is used in the solar tracking system. It has a combination of adjustable tilt angle and azimuth solar tracking system. A small tilt angle can adjust in every month is provide the same focus point. The entire dish concentrator system rotates from east to west to follow the solar azimuth.

The entire system is rotated by the 120 teeth gear wheel arrangement, which is driven by the 16 teeth small gear wheel. The driving gear ' 1 ' is connected with stepper motor and it will drive the gear ' 2 '. The gear ratio between the drive and driven wheel is 7.5:1.A manual mechanical tilt arrangement is provided in addition to the tracking arrangement for monthly creeping movement of the sun. The driven wheel coupled with a cylindrical pipe and this entire set up is inserted into the polished central axis rod and it allows clear movement of the dish. An azimuth action will be done only by the driven gear.

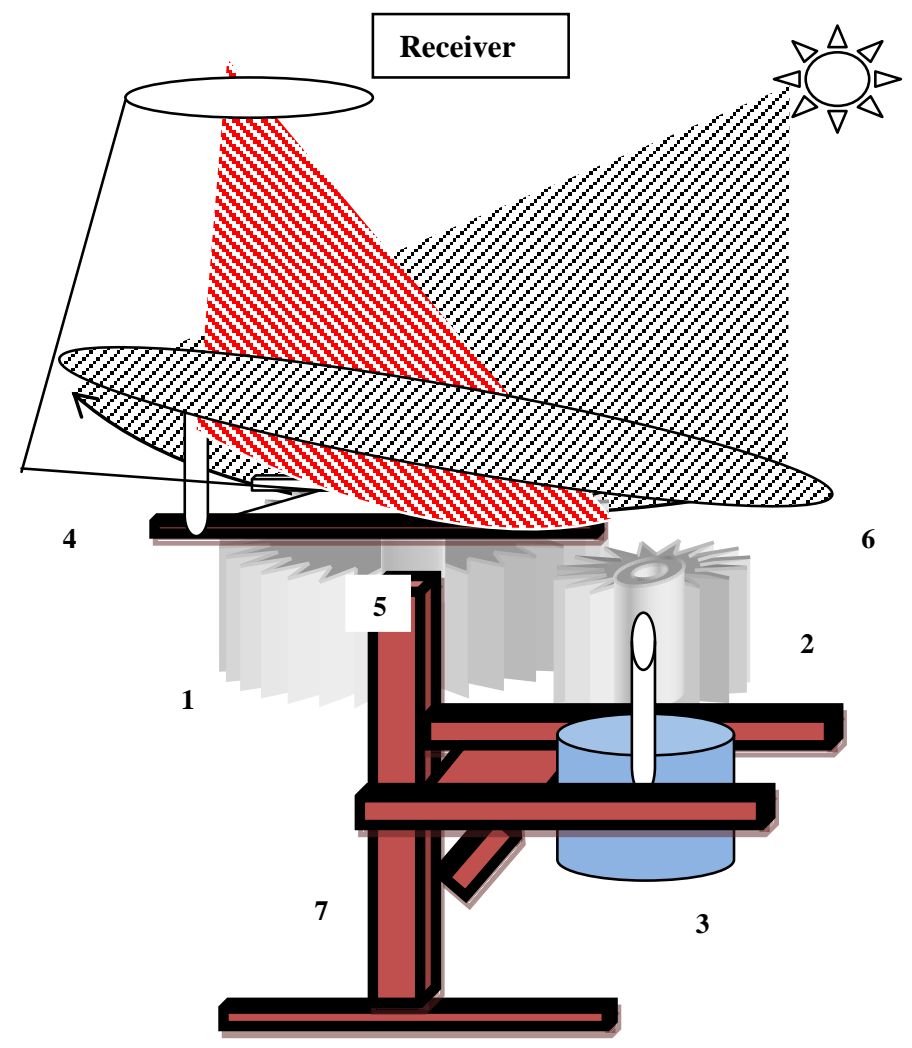

Fig.3. Mechanical Design 
Fig. 3, number 1 is represented driven gear, 2 and 3 are represents driving gear \& stepper motor respectively. The vertical rod marked Number 4, is used to adjust the tilt alignment. The cylindrical steel pipe with Number 5 is the base plate holding the concentrator. Number 6 corresponds to solar concentrator \& vertical pipe number 7 is a supporting structure. Initially, the complete set up is placed towards the east at the fixed zenith angle and it changes periodically, every month. The tracking system was designed to orient the concentrator towards the sun's position and it is a regularly required process. It allows the concentrator to receive the maximum incoming solar energy. In this method of tracking accurate stepping movement is obtained based on RTC (Real Time Clock) which drives the software. The timing pulses from RTC form the basis of tracking forming the bottom layer and the overall software is a layer above it.

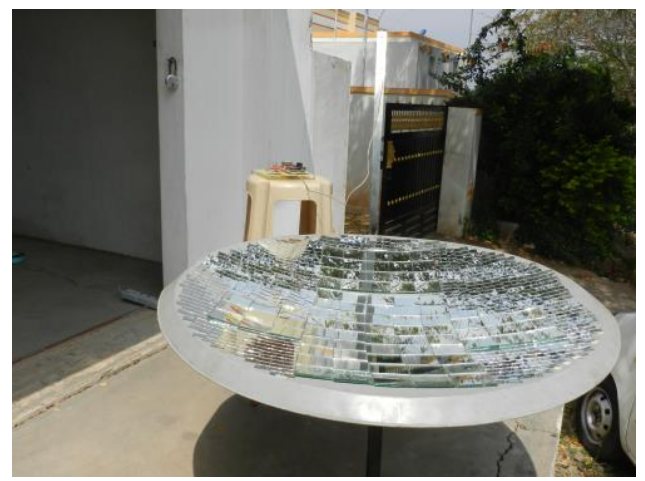

Fig.4. Entire Tracking System

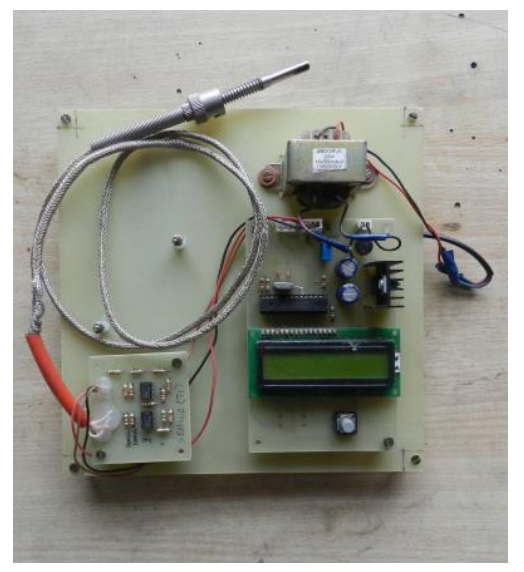

Fig.5. Temperature Measurement Unit

The device tracking starts from 10.00 AM and this motion is activated at the predefined time interval. The performance of the tracking is increased by changing the different angle and time interval between every an hour. The system starts to follow the sun at 10.00 AM to 3.00 PM. The solar concentrator has programmed to rotate every $2 \mathrm{~min}$ at an angle of $7.2^{\circ}$ from $10.00 \mathrm{AM}$ to $11.00 \mathrm{AM}$ and every $4 \mathrm{~min}$ at an angle of $3.6^{\circ}$ from 11.00 AM to 1.00 PM. The system again rotates with 2 min time interval between $1.00 \mathrm{PM}$ to $3.00 \mathrm{PM}$ at an angle of $7.2^{\circ}$. Fig. 4 shows the whole tracking system. The tracking system will orient the concentrator for diverting the solar irradiation to the receiver. The shape of the receiver was elliptical, so it can observe the solar radiation in the best possible way for the proposed concentrator design. The collected thermal energy has 
measured using K-type thermocouple sensor and thermometer gun. Fig. 5 is the temperature measurement unit. The temperature values recorded as data is continuously sent to the computer at regular time intervals.

\section{Results and Discussion}

The operation of the experimental arrangement was verified and temperature data were recorded. The temperature at the receiver point was recorded daily in specified time. K-type and thermometer gun were used to measure the obtained temperature from the concentrator (K-type thermocouple temperature sensor is the combination of chromium and aluminum and with temperature range of -200 to $1350{ }^{\circ} \mathrm{C}$ and sensitivity of $0.041 \mathrm{mv}$ ). The infrared thermometer gun (model-BEETECH-MT4) used to measure the temperature in the range from -50 to $550{ }^{\circ} \mathrm{C} /-58{ }^{\circ} \mathrm{F}-1022^{\circ} \mathrm{F}$.

The infrared thermometer measurement ratio of distance to spot size (D:S) is 12:1. The received temperature data was transmitted using GSM wireless unit from the remote area to monitoring unit. In monitoring area, the computer receives temperature values and maintains record regularly. The temperature value of $342{ }^{\circ} \mathrm{C}$ was noted during measurement of temperature using thermometer gun, which is the maximum value recorded. RTC (Real Time Clock) with open loop programming method gives an accurate data for aligning the solar concentrator at the sun's position. The temperature of the receiver increases until it reaches the maximum temperature up to noon and decreases on sunset. The graphical representation was drawn with recorded values shows the favorable result of phenomenal heating. Fig. 6 and Fig. 7 show the received temperature and ambient temperature recorded at the time of performing the experiment. The mechanical design and programming software were tested and gave appropriate results, as could be observed by the temperature values recorded. The practical experiments performed to examine the efficiency of simple RTC based open loop dual axis solar tracking system resulted in successful implementation of a new technique in tracking method. The received temperature data were sent to GSM module and it is also stored in the computer as data logger.

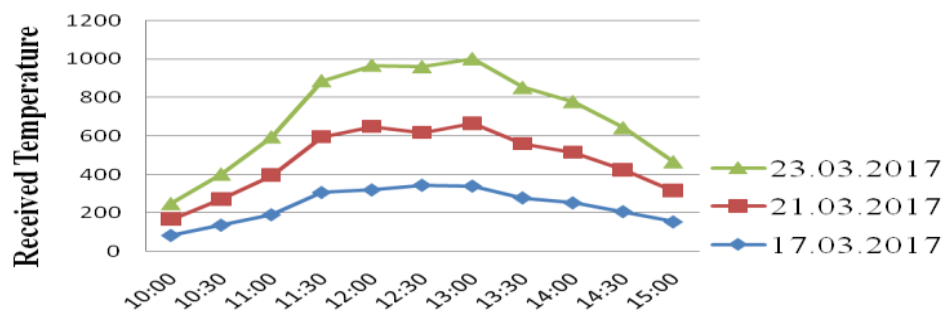

Time (hr)

Fig.6. Received Temperature Variation

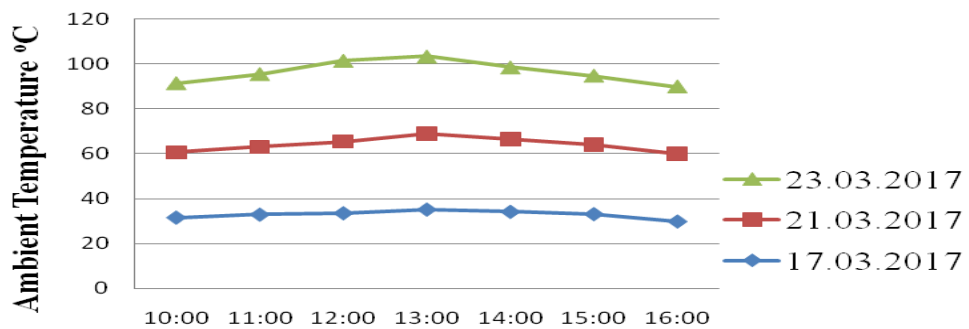

Time (hr)

Fig.7. Received Ambient Temperature 


\section{Conclusion}

The automated and tested dual axis solar tracking system for innovatively designed model novel solar concentrator showed good performance and outcome. Since RTC (Real Time Clock) was used for solar tracking, it gave an appropriate tracking data in a real time. The accuracy of this solar tracking system is found to be better than the sensor based tracking system. The advantage in RTC based tracking method is its independence to the geographical topography and other environmental conditions. Various programming functions are used in software and so, errors in tracking are drastically reduced. Compared to other solar tracking systems, the RTC based solar tracking system consumes least power. The AOCCC system produces a maximum temperature of $342{ }^{\circ} \mathrm{C}$ for normal experimental conditions. The tracking system used for the concentrator is also very portable.

\section{Acknowledgments}

The authors wishes to thank the technical support from OMTRONICS LED LIGHTING SOLUTIONS, Coimbatore, TamilNadu and M/s THRIVE TECHNOLOGY, Coimbatore, TamilNadu, India which lead to designing the solar concentrator along with tracking system, perform observations and test the system.

\section{References}

[1] Yi Ma, Guihua Li and Runsheng Tang.. "Optical performance of vertical axis three azimuth angles tracked solar panels". Applied Energy-2011. 88:1784-1791.

[2] Mohammed S. Al-Sou, Essam Abdallah, Ali Akayleh, Salah Abdallah and Eyad S. Hrayshat. "A parabolic solar cooker with automatic two axes sun tracking system". Applied Energy-2010. 87: 463-470.

[3] Keong Chong, Chee-Woon Wong, "General Formula for On-Axis Sun-Tracking System", Solar Collectors and Panels, Theory and Applications. October 2010.

[4] Riyad Abu-Malouh, Salah Abdallah and Iyad M. Muslih. "Design, construction and operation of spherical solar cooker with automatic sun tracking system". Energy Conversion and Management-2011. 52:615620.

[5] M. Abouzeid, Use of a reluctance stepper motor for solar tracking based on a programmable logic array (PLA) controller, Renewable Energy -2001.23. 551-560.

[6] Tsung-Chieh Cheng, Wei-Cheng Hung, and Te-Hua Fang. "Two-Axis Solar Heat Collection Tracker System for Solar Thermal Applications". International Journal of Photoenergy-2013. Article ID 803457.

[7] M Xiaoshan Jin, Guoqiang Xu, Rongjiu Zhou, Xiang Luo and Yongkai Quan. "A Sun Tracking System Design for a Large Dish Solar Concentrator". International Journal of Clean Coal and Energy-2013. 2: 1620.

[8] H. Arbab, B. Jazi and M. Rezagholizadeh. "A computer tracking system of solar dish with based on picture processing of bar shadow, Renewable Energy-2009.34: 1114-1118.

[9] Basil M. Hamed, Mohammed S. El-Moghany, "Fuzzy Controller Design using FPGA for Sun Tracking in Solar Array System", I.J. Intelligent Systems and Applications, 2012, 1, 46-52.

[10] Salihu O. Aliyu, Michael Okwori and Elizabeth N. Onwuka, "A Prototype Automatic Solar Panel Controller (ASPC) with Night-time Hibernation”, I.J. Intelligent Systems and Applications, 2016, 8, 1825.

[11] M.T. Makhloufi, M.S. Khireddine, Y. Abdessemed, A. Boutarfa, "Tracking Power Photovoltaic System using Artificial Neural Network Control Strategy", I.J. Intelligent Systems and Applications, 2014, 12, 1726. 


\section{Authors' Profiles}

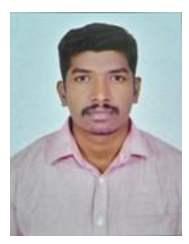

Krishna Kumar.N received his B.Sc and M.Sc. degree from the Electronics Department at PSG College of Arts and Science, Coimbatore, TamilNadu, India (Affiliated to Bharathiyar University) in 2006 and 2013 respectively. He has pursuing Ph.D in electronics at the same university from 2013. He has worked as Associate Supervisor from 2006 to 2008 at Larsen \& Toubro, Mysore, Karnataka, Inida in the Department of Energy Meter. He has worked as Embedded Engineer at Thrive Technology, Coimbatore, TamilNadu, India from 2008 to 2011.

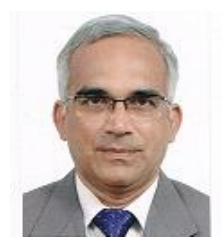

Dr. Venkat Subramaniam received his Master's degree and Ph.D. degree from the Physics Department at Bharathiyar University in 1986 and 2011, respectively. He has been Professor from 1987 to till date in the Department of Electronics at PSG College of Arts and Science, Coimbatore, Tamil Nadu, India. He has served as chairman, Board of Examiners for $\mathrm{PhD} / \mathrm{Mphil}$ and PG at Bharathiar University, Coimbatore and Bharathidasan University, Trichy respectively. He has served as member, Board of Examiners for Madurai Kamaraj University,Madurai and Periyar University,Salem. He has served as Vidyalaya Management Committee Member (twice), Kendriya Vidyalaya, HRD Ministry, Govt. of India.

How to cite this paper: Krishna Kumar.N, Venkat Subramaniam,"An Automatic Dual Axis Sun Tracker and Temperature Monitoring System for Novel Solar Dish Concentrator", International Journal of Engineering and Manufacturing(IJEM), Vol.8, No.1, pp.16-24, 2018.DOI: 10.5815/ijem.2018.01.02 